\title{
Associated factors to the consumption of ultra-processed foods and its relation with dietary sources in Portugal
}

\author{
Vânia Magalhães ${ }^{1,2} *\left(\mathbb{D}\right.$, Milton Severo ${ }^{1,2}$, Daniela Correia ${ }^{1,2}$, Duarte Torres ${ }^{1,3}$, \\ Renata Costa de Miranda ${ }^{4,5}$ (D) Fernanda Rauber ${ }^{4,6}$, Renata Levy ${ }^{4,5}$, Sara Rodrigues ${ }^{1,3}$ (1) and Carla Lopes ${ }^{1,2}$ \\ ${ }^{1}$ EPIUnit - Instituto de Saúde Pública da Universidade do Porto, Rua das Taipas, $n^{\circ} 135$, 4050-600 Porto, Portugal \\ ${ }^{2}$ Departamento de Ciências da Saúde Pública e Forenses e Educação Médica, Faculdade de Medicina da Universidade do Porto, Alameda Professor \\ Hernâni Monteiro, 4200-319 Porto, Portugal \\ ${ }^{3}$ Faculdade de Ciências da Nutrição e Alimentação da Universidade do Porto, Rua do Campo Alegre, n 823, 4150-180 Porto, Portugal \\ ${ }^{4}$ Núcleo de Pesquisas Epidemiológicas em Nutrição e Saúde, Universidade de São Paulo, São Paulo 01246-904, Bražil \\ ${ }^{5}$ Departamento de Medicina Preventiva, Escola de Medicina, Universidade de São Paulo, São Paulo 01246-903, Brazil \\ ${ }^{6}$ Departamento de Nutrição, Escola de Saúde Pública, Universidade de São Paulo, São Paulo 01246-904, Brazil
}

(Received 11 February 2021 - Final revision received 13 July 2021 - Accepted 30 July 2021)

Journal of Nutritional Science (2021), vol. 10, e89, page 1 of 11

doi: $10.1017 /$ jns.2021.61

Abstract

Ultra-processed foods (UPFs) are common worldwide and associated with poorer health outcomes. This work aimed to explore the UPF consumption associated factors and its main dietary sources, by sex, in Portugal. Participants from the National Food, Nutrition and Physical Activity Survey (IAN-AF) 2015-2016, aged 3-84 years, were included ( $n$ 5005). Dietary intake was assessed through two 1-day food diaries/24 h recalls. UPFs were identified using the NOVA classification. Associations were evaluated through linear regression models. Median UPF consumption was $257 \mathrm{~g} / \mathrm{d}(10 \cdot 6 \% \mathrm{of}$ total quantity; $23.8 \%$ of total energy). Adolescents were those with higher consumption $(490 \mathrm{~g} / \mathrm{d})$. Compared to adults, younger ages were positively associated with UPF consumption (e.g. adolescents ( $\hat{\beta}$-females: 192, $95 \%$ confidence interval (CI): 135, 249; $\hat{\beta}$-males: 327, $95 \%$ CI: 277, 377)). A lower educational level was associated with lower UPF consumption ( $\hat{\beta}$-females: $-63 ; 95 \% \mathrm{CI}:-91,-34 ; \hat{\beta}$-males: $-68 ; 95 \% \mathrm{CI}:-124,-12)$. Also, a lower UPF consumption was observed in married males/couples compared to singles $(\hat{\beta}:-48,95 \% \mathrm{CI}:-96,-1)$. Furthermore, female current/former smokers were associated with a higher UPF consumption $v$. never smokers $(\hat{\beta}: 79,95 \%$ CI: 41, $118 ; \hat{\beta}: 42,95 \% \mathrm{CI}: 8,75$, respectively). Main UPF sources were yoghurts, soft drinks and cold meats/sausages differing strongly by sex, age and education level. Yoghurts containing additives were the main contributors to the UPF consumption in children and adult females from all education ( 20\%). Soft drinks were leaders in adolescents (females: $26 \cdot 0 \%$; males: $31 \cdot 6 \%$ ) and young male adults $(24.4 \%)$. Cold meats/sausages stood out among low-educated males $(20 \cdot 5 \%)$. Males, younger age groups, higher education, children with less-educated parents, married/couple males and smoking females were positively associated with UPF consumption.

Key words: National survey: Nova: Portugal: Ultra-processed foods

\section{Introduction}

In the recent years, global changes in eating patterns have been observed, notably the increase of ultra-processed foods (UPF) consumption ${ }^{(1,2)}$. According to Monteiro et al., the UPF manufacture includes the fractioning of whole foods into substances, chemical modifications of these substances, assembly of unmodified and modified food substances, frequent use of cosmetic additives and sophisticated packaging ${ }^{(3)}$. The appearance and growth of these products were due to both economic and social pressure. Ready-to-eat and ready-to-heat food products have become attractive options as societies become more urbanised, incomes have increased

* Corresponding author: Vânia Magalhães, email vania.magalhaes@ispup.up.pt

(C) The Author(s), 2021. Published by Cambridge University Press on behalf of The Nutrition Society. This is an Open Access article, distributed under the terms of the Creative Commons Attribution licence (http://creativecommons.org/licenses/by/4.0/), which permits unrestricted re-use, distribution, and reproduction in any medium, provided the original work is properly cited. 
and the proportion of women employed outside the home has increased $^{(4)}$. The fast movement is consolidated as a consequence of the scarcity of time ${ }^{(5)}$ and the globalised world favours the mass production and consumption of convenience foods. Globally, bakery products (such as cakes, sweets and industrial breads) and soft drinks have been identified as the main contributors to the UPF sales volume ${ }^{(6)}$. However, other food groups are relevant depending on the region, such as dairy products, processed fruits and vegetables, or baked goods ${ }^{(6)}$.

Data from the US National Health and Nutrition Examination Survey 2011-2016 ${ }^{(7)}$ showed that UPF contributed to $55.4 \%$ of energy intake in adults. Similar results were found in the UK National Diet and Nutrition Survey 20082014 describing 56.8\% UPF contribution to total energy intake for total population ${ }^{(8)}$. Moreover, a recent study in Portuguese adults and elderly ${ }^{(9)}$ found a dietary share of 23.8 and $16.0 \%$, respectively. This study has also shown that as UPF consumption increases, energy and carbohydrate intake also increases, while fibre, sodium and potassium intake decreases. The negative impact of UPF consumption on health has been systematically reviewed. Its high consumption has already been associated with overweight and obesity ${ }^{(10-13)}$, other cardio metabolic risk-related outcomes ${ }^{(12-14)}$, some types of cancer, depression, frailty ${ }^{(13)}$ and even mortality ${ }^{(11)}$.

To better support the development of food policies adapted to the needs of each population, it is important to identify which factors are associated with UPF consumption and which are its main dietary sources. Worldwide, few studies have assessed the association between sociodemographic and behavioural factors and UPF consumption. None, to our knowledge, has assessed its relationship with its main dietary sources. Studies in high-income countries have reported an inverse association between the consumption of UPF and markers of socio-economic position, ${ }^{(5,15,16)}$ but the opposite has been reported in Brazil $^{(17)}$ and Colombia ${ }^{(18)}$. In Portugal ${ }^{(19)}$, compared to those having a lower socio-economic status, children and adolescents belonging to a higher socio-economic status had a higher daily intake of fruit and vegetables, white meat, fish and eggs, with no association being observed for sweets and soft drinks. However, a positive association between socio-economic status and the consumption of salty snacks was found only in adolescents, which reinforce the interest in studying UPF consumption in each age group. Moreover, the aggregation of unhealthy behaviours has been described, so that the coexistence of high UPF consumption with other unhealthy behaviours deserves to be studied.

In UK adults, similar UPF consumption was observed in subjects with different physical activity levels ${ }^{(20)}$. However, among Brazilian adolescents, longer time spent in sedentary behaviours was associated with a higher prevalence of UPF consumption emphasising the need for integrated health interventions ${ }^{(21)}$. Thus, the aim of the present study was to explore the associated sociodemographic and behavioural factors as well as the main dietary sources of UPF consumption in Portugal, by sex, using individual dietary data from a national survey. Sociodemographic factors including age, education, region, urbanisation level, civil status, household members and food insecurity were considered, while behavioural factors included physical activity and smoking status.

\section{Methodology}

\section{Survey design and participants}

Data from the National Food, Nutrition and Physical Activity Survey, IAN-AF, 2015-2016, were used. Details on the study design have been published previously ${ }^{(22,23)}$. A representative sample of the Portuguese general population, aged between 3 months and 84 years of age, was selected from the National Health Registry, by multistage sampling, in each geographical region (NUT II). The study was approved by the National Commission for Data Protection, the Ethical Committee of the Institute of Public Health of the University of Porto and from the Ethical Commissions of each of the Regional Administrations of Health. A written informed consent was obtained from all participants. For children, the informed consent was signed by parents or legal caregivers. A total of 5811 participants completed two computer-assisted face-to-face interviews conducted by trained nutritionists at the primary health care units or participants' homes. The response rate among eligible participants was $35.0 \%$, higher in children and adolescents (approximately $46 \%$ ) and lower in the elderly (approximately $20 \%$ ). Assuming strong differences in food patterns, in the present study, participants aged less than 3 years were excluded, and the final sample was left with 5005 participants.

\section{Data collection}

The data collection followed the guidelines of the pan-European food consumption survey (EU-Menu), previously described $^{(24)}$. The information was collected by the interviewer using face-to-face questionnaires and directly handled in the You eAt\&Move, an e-platform specifically designed for the IAN-AF ${ }^{(23,25)}$.

\section{Dietary variables}

To account for seasonal variability, fieldwork was carried out within 12 months. Dietary intake was obtained by two nonconsecutive food diaries in the case of children under the age of 10 years, or by two $24 \mathrm{~h}$ dietary recalls (8-15 days apart) for the remaining age groups ${ }^{(26)}$. The eAT24 module from the e-platform integrates the harmonised food classification and description system EFSA FoodEx $2^{(27)}$ and the Portuguese food composition table ${ }^{(28)}$ complemented with nutritional data from other countries (1777 food items). Foods were grouped into fifteen food groups and seventy-five sub-groups, according to nutritional similarities, source and dietary use ${ }^{(29)}$. The eAT24 also includes several food quantification methods, such as an electronic picture book ${ }^{(30)}$, household measures, standard units, volume, weight and default portion. As a quality control procedure, at the end of each interview, energy and nutrient estimates were briefly analysed by the interviewer. Dietary data collected using the eAT24 software were previously 
validated in a subsample using urinary biomarkers ${ }^{(25)}$ Pearson's correlation coefficients for protein, potassium and sodium were $0.33,0.64$ and $0 \cdot 26$, respectively.

Food classification according to the processing degree and purpose

All reported items were classed according to the NOVA classification $^{(3,31)}$. NOVA is a food classification system based on the degree and purpose of food processing developed by researchers at the University of São Paulo, Brazil. It classifies all foods into four groups: (1) unprocessed and minimally processed foods, (2) processed culinary ingredients, (3) processed foods and (4) UPF.

The detailed information on food intake obtained in the dietary assessment method used made it possible to classify foods from the same food group differently according to the specific characteristics of each. For example, unsweetened plain yoghurts were classified as 1, plain yoghurts with added sugar as 3 and flavoured yoghurts or with other cosmetic additives as 4 .

In order to classify at the food level, recipes were previously disaggregated into ingredients. Capsule-type supplements were not included in this classification. Coding according to the degree and purpose of food processing was conducted independently by two researchers (from Portugal and Brazil). Subsequently, both lists were verified by a third researcher who identified discrepant items, later discussed among all teams and classified by consensus. In case of doubtful classification, the experts decided on the most conservative classification, the one corresponding to the lowest processing level.

\section{Non-dietary variables}

Data on sociodemographic characteristics, food security and health behaviours were collected. Based on the address of the participants, the urbanisation level was assigned - predominantly urban area, medially urban area and predominantly rural area - following the classification proposed by the Portuguese National Institute of Statistics ${ }^{(32)}$. The education level was recoded into equal or less than 6 years of schooling, 7 to12 years, or more than 12 years. For those aged less than 18 years, the parents' highest education was considered. Data on household food insecurity were obtained for adults by applying a slightly modified questionnaire developed by Bickel et al. ${ }^{(33)}$, as previously described ${ }^{(22)}$. Participants with moderate insecurity were combined with those with severe insecurity in order to analyse food security $v$. food insecurity.

Physical activity was accessed by the International Physical Activity Questionnaire (IPAQ) short-form ${ }^{(34)}$ in those aged 15 and above. Each participant was classified as active, minimally active or inactive.

In terms of smoking habit, each participant aged 15 or over was rated for tobacco use. Three classes were used: 'never smoked', 'former smoker' (those who smoked but currently no longer smoke) and 'current smoker'. In the case of adolescents, only information was collected on whether they have never smoked or currently smoking, being not possible to identify former smokers.

\section{Statistical analysis}

Descriptive data of the absolute usual intake of UPF and non-UPF (in quantity $-\mathrm{g}$ ) as well as of the relative contribution of UPF to the total quantity of food (\% TQ) were studied through the analysis of its distribution. The usual intake distributions were obtained using the Statistical Program to Assess Dietary Exposure (SPADE) software ${ }^{(35)}$. Briefly, this software applies the following steps: (1) Box-Cox transformation (transforming observed food consumption results into a symmetrical distribution); (2) modelling daily food consumption as a function of age (using fractional polynomials in order to estimate intra- and inter-individual variance using a linear random-effects model); (3) re-transformation to the original scale (based on solving an integral using the Gaussian quadrature method and the parameter estimates of the previous step).

Probabilistic weights were used to achieve representativeness of the Portuguese population, in order to compensate for oversampling of regions and age groups. Moreover, the use of the probabilistic weights accounts for the cluster effect of primary sample units (primary health care units) and the stratification by the region (NUT II).

In the study of associated factors, UPFs were considered as absolute quantity instead of a proportion for the following reasons. First, when estimating a proportion of the quantity of UPF to the total quantity of foods consumed, it is assumed that UPF and total food consumption are directly proportional on a logarithmic scale (Supplementary Figure S1). This mathematical assumption was checked, and it was noticed that, in this case, the consumption of UPF grows as a function of the square root of the total quantity of food $(\hat{\beta}: 0 \cdot 486,95 \%$ CI: $0.364,0.607)$. Second, the predictive capacity of that model proved to be low $\left(R^{2} 0.009\right)$ that is, even correcting the power of the denominator, the total quantity explains only $0.9 \%$ of the UPF quantity variance. In addition, we found weak correlations between the quantity of UPF and the total food quantity $(\rho \quad 0 \cdot 16)$ and between the quantity of UPF and the quantity of non-UPF $(\rho-0 \cdot 17)$. It should be noted that other authors ${ }^{(36)}$ already warned that choosing relative approaches may create mathematical dependency between the numerator and the denominator, introducing residual confounding.

Linear associations between sociodemographic and behavioural factors and the quantity of UPF consumption based on 2-d mean intake were evaluated through linear regression coefficients $(\hat{\beta})$ and the respective $95 \%$ CI. The 2 -d mean intake is more symmetric than the usual mean in which the 2 days for each individual are used. The residuals of the models showed a reasonable skewness, around $+1 \cdot 5$, which is acceptable for large samples, as it is between -2 and $+2^{(37)}$. In addition, the histogram and the Q-Q graph were checked, with a small deviation being observed, which allowed for the use of linear regression models.

According to the literature, sex seems to have a modifying effect on several of the studied exposures. Therefore, the effect of sex on the relationship of each of the other variables studied with the consumption of UPF was evaluated and several significant results were found $(P<0 \cdot 001)$. For this reason, 
the analysis of associated factors was stratified by sex. The modifying effects were tested using nested models with and without interaction, observing the significance of the interaction using ANOVA with Rao-Scott LRT. In addition to the crude model, an adjusted model for age group and education level (Model 1) was assessed, as well as a model with an additional adjustment for the remaining quantity of foods consumed (non-UPF) (Model 2).

A significance level of 0.05 was considered. Statistical analyses were carried out using $\mathrm{R}$ software ${ }^{(38)}$, version 3.6.1 for Windows. Probabilistic weights were applied using the package 'survey' ${ }^{(39)}$, according to the complex sampling design.

\section{Results}

Table 1 shows the main characteristics of the sample and describes its UPF consumption. In the Portuguese population aged between 3 and 84 years, the median UPF consumption was $257 \mathrm{~g} / \mathrm{d}$, which corresponds to $10.6 \% \mathrm{TQ}$ (454 kcal/d; $23 \cdot 8$ of the total energy intake ( $\%$ TEI - data not shown). The absolute quantity consumed seems to be higher in males (263 v. $254 \mathrm{~g} / \mathrm{d}$ in females), although the relative consumption to the total food quantity was higher in females (11.4 v. $9.9 \%$ TQ in males). Regarding the age groups, the absolute consumed quantity seems to be higher in adolescents, reaching $490 \mathrm{~g} / \mathrm{d}$, while analysing the relative contribution it seems to be higher in children, tending to decrease with increasing age (children: 22.3\%TQ; adolescents: $21.6 \% \mathrm{TQ}$; younger adults: $13.4 \%$ TQ; adults: $7 \cdot 9 \%$ TQ; elderly: $5.4 \%$ TQ). For the geographical regions, the UPF consumption seems to be higher in the Autonomous Region of Azores (an archipelago) and in the Metropolitan Area of Lisbon (capital), counting on 300 and $296 \mathrm{~g} / \mathrm{d}$, respectively. It should also be noted that the Autonomous Region of Madeira showed to have the lowest absolute UPF consumption (237 g/d). However, as it also presented one of the lowest non-UFF consumptions, the contribution of UPF to the total quantity of food consumed was higher, which reinforces the use of the absolute quantity as a measure of UPF assessment to avoid residual confounding. Individuals with less than 6 years of education showed the lowest UPF consumption $(209 \mathrm{~g} / \mathrm{d}$ ) and also the lowest non-UPF consumption (2075 g/d). Higher educated participants were those with the smallest UPF contribution to the total quantity since they consume more UPF but also more non-UPF. Higher UPF consumption was observed for current smokers $(274 \mathrm{~g} / \mathrm{d} v$. never smoked: $231 \mathrm{~g} / \mathrm{d}$; former smoker: $236 \mathrm{~g} / \mathrm{d}$ ). However, former smokers were those who consumed more non-UPF (never smoked: $2124 \mathrm{~g} / \mathrm{d} v$. former smokers: $2393 \mathrm{~g} / \mathrm{d} v$. current smoker: $2257 \mathrm{~g} / \mathrm{d}$ ). For the remaining studied variables, no appreciable differences were found for descriptive purposes.

Table 2 shows the association between sociodemographic and behavioural characteristics and the absolute quantity of UPF by sex. After adjustment for education and non-UPF consumption (Model 2), considering older adults as a reference, all lower age groups were positively associated with UPF consumption in males and females. The magnitude was higher in adolescents and different by $\operatorname{sex}(\hat{\beta}$ females:
192, $95 \%$ CI: 135, 249; $\hat{\beta}$ males: 327, $95 \%$ CI: 277,377$)$. Differences by sex were also observed in younger adults $(\hat{\beta}$ females: 100, $95 \%$ CI: 87, 133; $\hat{\beta}$ males: $235,95 \%$ CI: 190 , 280). Elderly individuals showed a significantly lower consumption of UPF than adults ( $\hat{\beta}$ females: $-63,95 \% \mathrm{CI}$ : $-91,-34 ; \hat{\beta}$ male: $-51,95 \%$ CI: $-93,-9)$. In both sex, the lowest level of education was associated with a lower consumption of UPF when compared to the highest level $(\hat{\beta}$ females: $-51,95 \% \mathrm{CI}:-86,-16 ; \hat{\beta}$ males: $-68,95 \% \mathrm{CI}$ : $-124,-12)$. A sensitivity analysis stratifying by age group (Supplementary Table S1) confirmed that this inverse association was only observed in adults $(\hat{\beta}$ younger adults: -117 ; $95 \% \mathrm{CI}:-197,-37 ; \hat{\beta}$ older adults: $-75 ; 95 \% \mathrm{CI}:-115$, $-34)$ and in children a lower level of education was associated with high consumption of UPF ( $\hat{\beta}$ children: $111 ; 95 \% \mathrm{CI}: 13$, 209). Furthermore, no significant association was observed in adolescent and elderly groups. Only in males, the consumption of UPF among those who were married/couples was lower when compared to singles ( $\hat{\beta}:-48,95 \% \mathrm{CI}:-96,-1)$. In females, being a current smoker or a former smoker was associated with a higher consumption of UPF compared to those who reported never having smoked $(\hat{\beta}$ current smoker: 79,95 $\%$ CI: 41, 118; $\hat{\beta}$ former smoker: $42,95 \% \mathrm{CI}: 8,75)$.

The top contributing food groups to the total quantity of UPF are presented in Fig. 1, for the different age groups, stratified by sex. Overall, in females, the leading contributor to the UPF quantity was yoghurts and other fermented milks containing cosmetic additives hereinafter described only as yoghurts $(20 \cdot 3 \%)$ and, in males, soft drinks $(17 \cdot 2$ $\%$ ) (data not shown). In both sex, children were the age group in which the three highest food contributors (yoghurts, flavoured milks and soft drinks) had more similar magnitude between them. In females, yoghurts showed the highest contribution in all age groups with the exception of adolescents, in which soft drinks led and whose difference to the second contributor (yoghurts) is notorious (26.0 v. $11.5 \%$, respectively). This gap between the first and second contributors was also observed in adolescent males (31.6 and $12.5 \%$, respectively). Soft drinks were still the main contributor to UPF consumption in young adult males $(24.4 \%)$. Cold meats and sausages take on greater importance in the oldest age groups of males (adults: $20.0 \%$; elderly: $17 \cdot 5 \%$ ). Only in the elderly, soft drinks were not among the main UPF contributors. On the other hand, the food group of cookies and biscuits emerged as a top contributor (females: 11.6\%; males: $10.7 \%$ ). Likewise, in Fig. 2, the top contributing food groups to the consumption of UPF are presented for the different education levels, stratified by sex. In females, regardless of education level, the main food contributor to the UPF was yoghurts. This food group deserves to be highlighted in the upper class since the contribution of the other groups was much lower $(21.5 \% v$. soft drinks: $9.2 \%$ and cold meats and sausages: $8.5 \%$ ). Cookies and biscuits showed a relevant contribution only in females with a lower level of education (10.9\%). On the other hand, in males, greater heterogeneity was observed in the food groups that mostly contributed to the consumption of UPF, according to the class of education. The contribution of yoghurts increased with the increase in schooling (from $9 \cdot 6$ to 
Table 1. Ultra-processed foods usual consumption according to sociodemographic and behavioural characteristics (weighted for the distribution of the Portuguese population)

\begin{tabular}{|c|c|c|c|c|c|}
\hline & \multirow{2}{*}{$n$} & \multirow{2}{*}{ Weighted \% } & \multirow{2}{*}{$\begin{array}{c}\text { Quantity of } \\
\text { UPF (g) } \\
\text { Median (P25-P75) }\end{array}$} & \multirow{2}{*}{$\begin{array}{c}\begin{array}{c}\text { Quantity of } \\
\text { non-UPF }(\mathrm{g})\end{array} \\
\text { Median (P25-P75) }\end{array}$} & \multirow{2}{*}{$\begin{array}{l}\% \text { of UPF to total } \\
\text { quantity }(\mathrm{g} / \mathrm{g}) \\
\text { Median (P25-P75) }\end{array}$} \\
\hline & & & & & \\
\hline & 5005 & 100 & $257(141-426)$ & $2176(1752-2645)$ & $10 \cdot 6(5 \cdot 9-17 \cdot 7)$ \\
\hline \multicolumn{6}{|l|}{ Sex } \\
\hline Female & 2613 & $51 \cdot 2$ & $254(151-393)$ & 1986 (1610-2392) & $11.4(6 \cdot 8-18 \cdot 0)$ \\
\hline Male & 2392 & $48 \cdot 8$ & $263(133-462)$ & 2412 (1962-2903) & $9.9(5 \cdot 0-17 \cdot 5)$ \\
\hline \multicolumn{6}{|l|}{ Age group } \\
\hline Children (3-9 years) & 521 & $5 \cdot 9$ & $414(273-595)$ & 1439 (1129-1798) & $22 \cdot 3(15 \cdot 5-30 \cdot 8)$ \\
\hline Adolescents (10-17 years) & 632 & $8 \cdot 1$ & $490(337-684)$ & $1841(1484-2247)$ & $21 \cdot 6(15 \cdot 0-30 \cdot 0)$ \\
\hline Younger adults (18-44 years) & 1758 & $38 \cdot 3$ & $340(217-503)$ & 2244 (1838-2701) & $13.4(8.5-19.9)$ \\
\hline Older adults (45-64 years) & 1344 & $29 \cdot 7$ & $195(115-306)$ & $2313(1904-2773)$ & $7 \cdot 9(4 \cdot 7-12 \cdot 3)$ \\
\hline Elderly (65-84 years) & 750 & $17 \cdot 9$ & $120(66-201)$ & $2171(1776-2616)$ & $5.4(3.0-8.9)$ \\
\hline \multicolumn{6}{|l|}{ Education } \\
\hline$\leq 6$ years & 1497 & $29 \cdot 7$ & $209(104-389)$ & 2075 (1668-2534) & $9.5(4.7-17.9)$ \\
\hline $7-12$ years & 2201 & $45 \cdot 8$ & $284(165-448)$ & $2221(1794-2690)$ & $11.5(6 \cdot 7-18.4)$ \\
\hline$>12$ years & 1291 & 24.5 & $284(174-428)$ & $2202(1791-2655)$ & $11.5(7 \cdot 0-17.5)$ \\
\hline \multicolumn{6}{|l|}{ Region } \\
\hline North & 838 & $35 \cdot 4$ & $242(129-409)$ & 2137 (1728-2589) & $10 \cdot 2(5 \cdot 5-17 \cdot 3)$ \\
\hline Centre & 886 & $21 \cdot 8$ & $249(143-400)$ & 2191 (1769-2659) & $10 \cdot 2(5 \cdot 9-16 \cdot 7)$ \\
\hline Lisbon Metropolitan Area & 701 & $26 \cdot 8$ & $296(167-477)$ & 2209 (1778-2678) & $12 \cdot 0(6 \cdot 9-19 \cdot 3)$ \\
\hline Alentejo & 575 & $6 \cdot 6$ & $268(147-447)$ & 2319 (1808-2894) & $10 \cdot 5(5 \cdot 7-17 \cdot 8)$ \\
\hline Algarve & 654 & $4 \cdot 3$ & $281(161-445)$ & 2401 (1955-2882) & $10 \cdot 4(5 \cdot 8-17 \cdot 1)$ \\
\hline Autonomous Region of Madeira & 683 & $2 \cdot 7$ & $237(128-396)$ & 1962 (1604-2362) & $11.5(6 \cdot 3-19 \cdot 1)$ \\
\hline Autonomous Region of Azores & 668 & 2.5 & $300(174-475)$ & 1893 (1493-2346) & $13.7(8 \cdot 0-21 \cdot 6)$ \\
\hline \multicolumn{6}{|l|}{ Urbanisation level } \\
\hline Predominantly urban area & 3650 & $77 \cdot 6$ & $262(144-431)$ & 2167 (1751-2625) & $10 \cdot 8(6 \cdot 0-17 \cdot 9)$ \\
\hline Medially urban area & 863 & $13 \cdot 8$ & $245(133-412)$ & $2182(1719-2711)$ & $9 \cdot 9(5 \cdot 3-17 \cdot 1)$ \\
\hline Predominantly rural area & 492 & 8.5 & $247(134-414)$ & $2172(1740-2648)$ & $10 \cdot 2(5 \cdot 6-17 \cdot 0)$ \\
\hline \multicolumn{6}{|l|}{ Civil status } \\
\hline Single, divorced or widowed & 1495 & 394 & $243(137-398)$ & $2234(1802-2717)$ & $9.9(5.8-15.9)$ \\
\hline Married, couples & 2354 & 606 & $225(118-388)$ & $2283(1880-2735)$ & $9 \cdot 0(4 \cdot 9-15 \cdot 1)$ \\
\hline \multicolumn{6}{|l|}{ Household members } \\
\hline $1-2$ & 1639 & $39 \cdot 9$ & $266(140-456)$ & $2181(1740-2659)$ & $10 \cdot 8(5 \cdot 8-18 \cdot 2)$ \\
\hline $3-4$ & 2752 & $54 \cdot 8$ & $255(135-425)$ & 2144 (1724-2613) & $10 \cdot 5(5 \cdot 7-17 \cdot 6)$ \\
\hline$\geq 5$ & 468 & $8 \cdot 3$ & $232(124-393)$ & 2075 (1668-2534) & $9 \cdot 8(5 \cdot 0-16 \cdot 9)$ \\
\hline \multicolumn{6}{|l|}{ Food insecurity } \\
\hline No & 3448 & $89 \cdot 9$ & $236(128-396)$ & 2277 (1868-2734) & $9 \cdot 4(5 \cdot 3-15 \cdot 5)$ \\
\hline Yes & 397 & $10 \cdot 1$ & $204(102-372)$ & $2283(1880-2735)$ & $9 \cdot 4(4 \cdot 8-16 \cdot 6)$ \\
\hline \multicolumn{6}{|l|}{ Physical activity level } \\
\hline Inactive & 1693 & $43 \cdot 2$ & $242(130-412)$ & $2142(1759-2563)$ & $10 \cdot 2(5 \cdot 6-17 \cdot 0)$ \\
\hline Minimally active & 1233 & $30 \cdot 3$ & $234(130-387)$ & 2240 (1825-2695) & $9.6(5 \cdot 4-15 \cdot 6)$ \\
\hline Active & 1022 & 6.5 & $236(124-405)$ & 2375 (1925-2887) & $9 \cdot 0(4 \cdot 9-15 \cdot 0)$ \\
\hline \multicolumn{6}{|l|}{ Smoking status } \\
\hline Never smoked & 1952 & $49 \cdot 4$ & $231(123-391)$ & 2124 (1734-2555) & $9 \cdot 8(5 \cdot 4-16 \cdot 1)$ \\
\hline Former smoker & 1157 & $30 \cdot 1$ & $236(126-402)$ & 2393 (1948-2892) & $9 \cdot 0(4 \cdot 8-15 \cdot 3)$ \\
\hline Current smoker & 831 & $20 \cdot 5$ & $274(151-460)$ & 2257 (1836-2729) & $11 \cdot 1(6 \cdot 4-18 \cdot 2)$ \\
\hline
\end{tabular}

UPF, ultra-processed foods.

$18.7 \%$ ), in contrast to that of cold meats and sausages, which tends to decrease (from 20.5 to $10.9 \%$ ). The contribution of soft drinks was higher in the intermediate class $(20 \cdot 4 \% v . \leq 6$ years: $12 \cdot 6 \%$; $>12$ years: $15 \cdot 9 \%$ ).

\section{Discussion}

The present study found an UPF consumption of $257 \mathrm{~g} / \mathrm{d}$ (10.6\%TQ; $454 \mathrm{kcal} / \mathrm{d} ; 23.8 \% \mathrm{TEI})$, using data from 2015/ 2016 of the Portuguese population aged between 3 and 84 years. In addition, we highlight the relevant role of sex, age and educational level, since these were the factors with a higher association with UPF consumption, influencing their food sources. The purchase of UPF in Portugal was estimated to provide $10 \%$ of the household total available dietary energy in the year $2000^{(40)}$. An increase in UPF consumption from 2000 to 2015/2016 may have occurred. However, the results of these studies cannot be directly compared since data from 2000 refer to household data, whereas the 2015/2016 data came from individual dietary survey. As such, Household Budget Surveys have some limitations with respect to individual consumption data, notably foods wasted and foods eaten while dining out, which were not accounted for. UPF consumption was lower in Portugal than in most countries where it has been studied for similar age groups. Data from European countries namely from the Belgian Food Consumption Survey 2014/2015 showed that UPF contribute $32.6 \%$ to total energy intake, ${ }^{(41)}$ while data from the UK 
Table 2. Association between sociodemographic and behavioural characteristics and ultra-processed foods usual consumption (g), stratified by sex (weighted for the distribution of the Portuguese population), using linear regression models

\begin{tabular}{|c|c|c|c|c|c|c|}
\hline & \multicolumn{3}{|c|}{ Females } & \multicolumn{3}{|c|}{ Males } \\
\hline & $\begin{array}{l}\text { Crude model } \\
\hat{\beta}(95 \% \mathrm{Cl})\end{array}$ & $\begin{array}{c}\text { Model } 1 \\
\hat{\beta}(95 \% \mathrm{Cl})\end{array}$ & $\begin{array}{c}\text { Model } 2 \\
\hat{\beta}(95 \% \mathrm{Cl})\end{array}$ & $\begin{array}{l}\text { Crude model } \\
\hat{\beta}(95 \% \mathrm{Cl})\end{array}$ & $\begin{array}{c}\text { Model } 1 \\
\hat{\beta}(95 \% \mathrm{Cl})\end{array}$ & $\begin{array}{c}\text { Model } 2 \\
\hat{\beta}(95 \% \mathrm{Cl})\end{array}$ \\
\hline \multicolumn{7}{|l|}{ Age group } \\
\hline Children (3-9 years) & $195(148,242)$ & $179(130,228)$ & $140(89,191)$ & $241(195,287)$ & $221(174,269)$ & $179(128,231)$ \\
\hline Adolescents (10-17 years) & $228(174,282)$ & $215(156,274)$ & $192(135,249)$ & $367(317,418)$ & $348(298,398)$ & $327(277,377)$ \\
\hline Younger adults (18-44 years) & $119(88,149)$ & $103(70,137)$ & $100(67,133)$ & $260(215,305)$ & $236(191,282)$ & $235(190,280)$ \\
\hline Older adults (45-64 years) & Ref. & Ref. & Ref. & Ref. & Ref. & Ref. \\
\hline Elderly (65-84 years) & $-80(-109,-51)$ & $-64(-93,-35)$ & $-63(-91,-34)$ & $-62(-100,-23)$ & $-41(-82,1)$ & $-51(-93,-9)$ \\
\hline \multicolumn{7}{|c|}{ 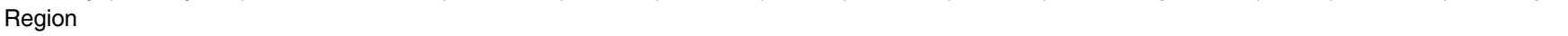 } \\
\hline North & Ref. & Ref. & Ref. & Ref. & Ref. & Ref. \\
\hline Centre & $-4(-35,28)$ & $4(-28,35)$ & $7(-26,40)$ & $-22(-72,29)$ & $-4(-53,45)$ & $0(-51,52)$ \\
\hline Lisbon Metropolitan Area & $47(7,87)$ & $39(-1,80)$ & $39(-3,81)$ & $85(24,146)$ & $70(17,124)$ & $76(19,133)$ \\
\hline Alentejo & $34(-10,77)$ & $46(9,83)$ & $50(9,90)$ & $-1(-65,63)$ & $30(-32,91)$ & $41(-23,106)$ \\
\hline Algarve & $34(-4,72)$ & $27(-5,60)$ & $36(1,70)$ & $21(-26,68)$ & $23(-22,68)$ & $32(-17,80)$ \\
\hline Autonomous Region of Madeira & $-14(-44,15)$ & $-13(-46,20)$ & $-23(-59,13)$ & $21(-33,75)$ & $5(-36,46)$ & $-7(-53,39)$ \\
\hline Autonomous Region of Azores & $62(15,110)$ & $50(7,93)$ & $40(-3,90)$ & $112(29,194)$ & $91(-12,171)$ & $82(-3,167)$ \\
\hline \multicolumn{7}{|l|}{ Education } \\
\hline$\leq 6$ years & $-136(-166,-105)$ & $-38(-74,-2)$ & $-51(-86,-16)$ & $-200(-251,-148)$ & $-64(-120,-9)$ & $-68(-124,-12)$ \\
\hline $7-12$ years & $11(-18,39)$ & $26(-1,54)$ & $21(-6,49)$ & $9(-35,53)$ & $12(-27,52)$ & $7(-32,46)$ \\
\hline$>12$ years & Ref. & Ref. & Ref. & Ref. & Ref. & Ref. \\
\hline \multicolumn{7}{|l|}{ Urbanisation level } \\
\hline Predominantly urban area & Ref. & Ref. & Ref. & Ref. & Ref. & Ref. \\
\hline Medially urban area & $-10(-40,19)$ & $-12(-46,23)$ & $-12(-49,24)$ & $-17(-71,37)$ & $-2(-47,44)$ & $1(-65,67)$ \\
\hline Predominantly rural area & $-16(-63,32)$ & $-21(-63,20)$ & $-21(-61,20)$ & $-33(-92,26)$ & $-2(-64,60)$ & $0(-47,48)$ \\
\hline \multicolumn{7}{|l|}{ Civil status } \\
\hline Single, divorced or widowed & Ref. & Ref. & Ref. & Ref. & Ref. & Ref. \\
\hline Married, couples & $-34(-64,-3)$ & $-14(-42,13)$ & $-10(-38,17)$ & $-144(-195,-92)$ & $-50(-98,-3)$ & $-48(-96,-1)$ \\
\hline \multicolumn{7}{|l|}{ Household members } \\
\hline $1-2$ & Ref. & Ref. & Ref. & Ref. & Ref. & Ref. \\
\hline $3-4$ & $94(64,124)$ & $-7(-37,24)$ & $-6(-37,25)$ & $138(95,181)$ & $15(-26,56)$ & $13(-29,54)$ \\
\hline$\geq 5$ & $72(22,122)$ & $-23(-75,28)$ & $-25(-79,29)$ & $178(101,254)$ & $7(-63,78)$ & $7(-63,77)$ \\
\hline \multicolumn{7}{|l|}{ Food insecurity } \\
\hline No & Ref. & Ref. & Ref. & Ref. & Ref. & Ref. \\
\hline Yes & $-36(-74,3)$ & $-7(-41,27)$ & $-11(-43,22)$ & $-64(-145,16)$ & $-32(-100,35)$ & $-43(-109,23)$ \\
\hline \multicolumn{7}{|l|}{ Physical activity level } \\
\hline Inactive & Ref. & Ref. & Ref. & Ref. & Ref. & Ref. \\
\hline Minimally active & $-25(-58,8)$ & $-26(-57,4)$ & $-24(-55,6)$ & $-13(-59,32)$ & $-17(-58,23)$ & $-11(-52,30)$ \\
\hline Active & $0(-40,40)$ & $0(-35,35)$ & $5(-31,40)$ & $47(-10,104)$ & $-5(-57,46)$ & $6(-48,60)$ \\
\hline \multicolumn{7}{|l|}{ Smoking status } \\
\hline Never smoked & Ref. & Ref. & Ref. & Ref. & Ref. & Ref. \\
\hline Former smoker & $68(35,102)$ & $35(2,68)$ & $42(8,75)$ & $-58(-106,-11)$ & $7(-32,46)$ & $12(-27,51)$ \\
\hline Current smoker & $128(82,174)$ & $83(45,121)$ & $79(41,118)$ & $-14(-68,40)$ & $-12(-61,37)$ & $-11(-60,39)$ \\
\hline
\end{tabular}

Model 1, adjusted for age group and education, Model 2, adjusted for age group, education and non-ultra-processed foods consumption. Bold denotes statistical significance ( $p$-value $<0.05)$.

National Diet and Nutrition Survey 2008-2014 pointed for $56 \cdot 8 \%{ }^{(8)}$. One of the possible justifications for the lower consumption in Portugal could be the adherence to traditional dietary patterns such as the Mediterranean Diet ${ }^{(42,43)}$ or the Southern European Atlantic Diet ${ }^{(44)}$. Recently, a study in the neighbouring country Spain showed that adherence to the traditional Mediterranean Diet was inversely associated with energy intake from $\mathrm{UPF}^{(45)}$. Portugal is a country that still preserves traditional eating habits based on fresh and minimally processed foods and culinary preparations made with these foods $^{(42)}$. In Spain, UPF accounted to $24.4 \%$ TEI among adults corresponding to $385 \mathrm{kcal} / \mathrm{d}^{(46)}$. Despite the differences in age groups between our study and the Spanish study, it is interesting to highlight that although the proportion in Spain is slightly higher than in Portugal (24.4 v. 23.8\%TEI), the absolute energy intake from UPF estimates is lower (385 $v$.
$454 \mathrm{kcal} / \mathrm{d}$ ), which suggests the need for a careful interpretation of the results when the proportion of TEI is expressed. We have also estimated the proportion of UPF to the total amount of food in order to take into account UPF that provides low or no energy (e.g. artificially sweetened beverages). Other previous studies have already analysed the consumption of UPF using the quantity proportion in order to account for low or no caloric food products. A study conducted on French adults (NutriNet-Santé) ${ }^{(47)}$ found a value lightly higher than ours $(17 \cdot 4 v \cdot 10 \cdot 6 \%$ TQ). This was an online study using convenience sampling from the general population, which may justify the differences in these geographically close countries.

In the present study, younger ages and higher education levels were significantly associated with higher UPF consumption. Due to the cross-sectional nature of this study, it is not possible to assess a potential cohort effect. However, it is 


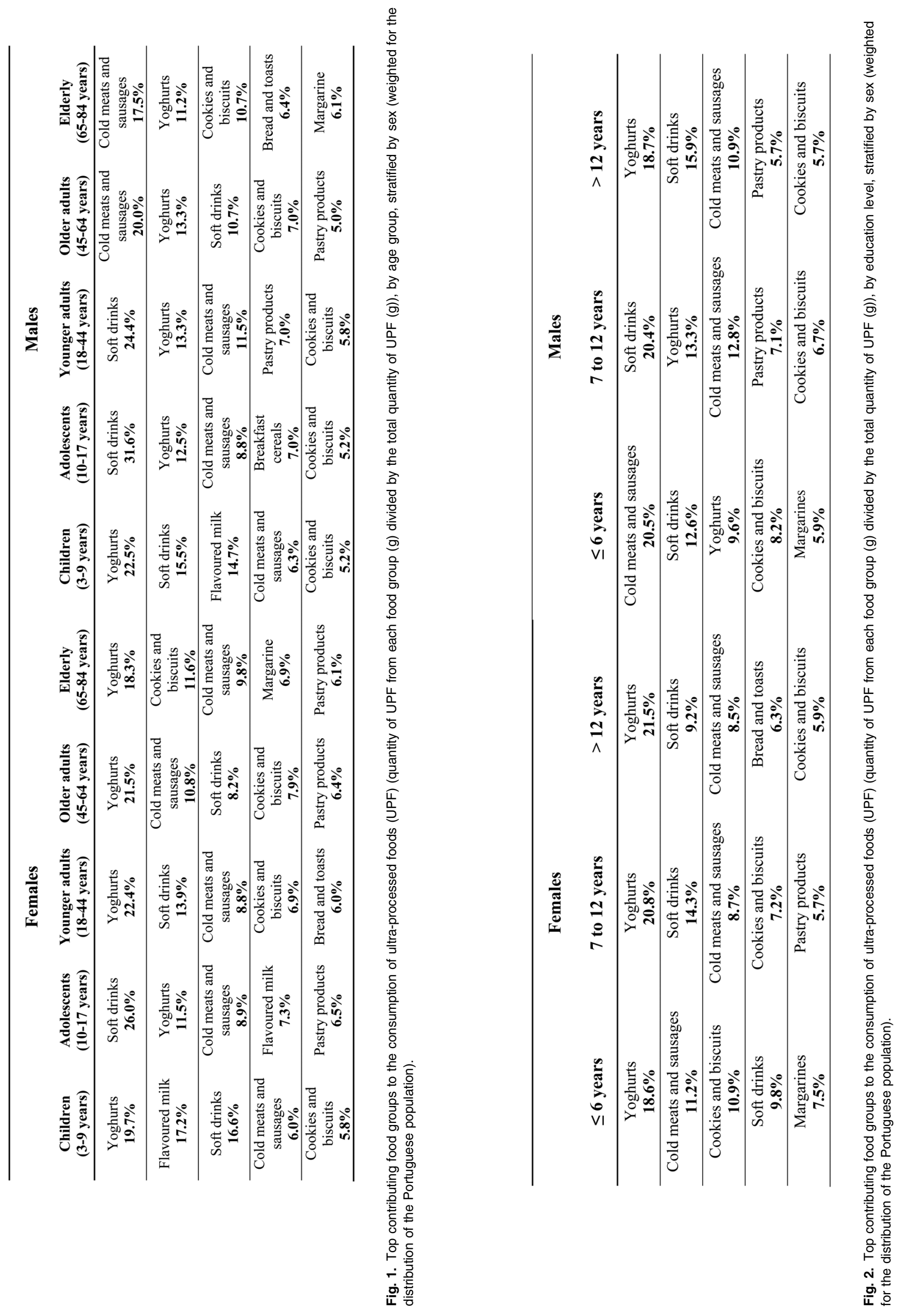


expected that the younger people from the current population could maintain these high levels in the future. These ideas are supported by tracking the effect of food patterns previously described during childhood ${ }^{(48,49)}$ and from adolescence into early adulthood ${ }^{(50,51)}$. Similar results were found in Mexico in 2012 where younger ages and medium and high socioeconomic status were also related to higher UPF consumption $^{(52)}$. In addition, urbanisation and living in the North of Mexico were also sociodemographic factors related to higher consumption of UPF. Similar findings were reported in Colombia ${ }^{(18)}$. On the other hand, in the USA, the UPF consumption decrease with the income level and with the level of education ${ }^{(53)}$. As countries become wealthy, its middle class will develop and tend to consume more UPF, typically produced by big multinational brands, maybe to exhibit socioeconomic status ${ }^{(1)}$. Moreover, in the present study, the different effects of the education level on UPF consumption observed by age can portray the nutritional transition over the generations. A study on the evolution of UPF prices over time in Brazil ${ }^{(54)}$ found that in 1995, UPFs were the most expensive food group. Since the early 2000s, prices of UPF underwent successive reductions, becoming cheaper and predictions point that in 2026 they will be even cheaper than minimally processed foods and culinary ingredients. With a lack of education in this matter, it is quite easy to be misled and to yield towards the marketing pressure. In Scotland, differences in exposure to food advertisements across areas with different socio-economic positions were observed, with higher exposures in low socio-economic areas ${ }^{(55)}$. In the present study, being a single male or a smoking female was shown to be associated with higher UPF consumption. Blanco-Rojo et al. reported a positive association between current smoking and UPF consumption in Spain ${ }^{(46)}$, as well as Rauber et al., in UK adults ${ }^{(20)}$. This positive association was also observed in the present study, suggesting an aggregation of these behaviours. Time doing sedentary activities (watching TV not included) and higher activity index were also associated with UPF consumption in Spain, as in the study of Rauber et al., but not in the present study. These differences can be explained by the use of different physical activity assessment methodologies and their respective categorisation as well as by a possible higher consumption of non-UPF in the higher classes of physical activity. Marital status has been less studied in this regard. In males, we found an inverse association between being married/couples and the consumption of UPF. We believe that this is due to eating more meals at home based on culinary preparations. In UK adults, better home food preparation skills and more frequent use of these skills tended to be associated with a lower UPF consumption ${ }^{(56)}$.

The present study was able to show that the top food groups contributing to UPF consumption were different by sex and according to the age group and education. In general, Portuguese individuals with a higher education level consumed higher quantity of UPF, with yoghurts being the main source, both in females and males. In the lower level of education, particularly in males, cold meats and sausages and soft drinks take on relevance. As the level of education increases, the consumption of cold meats and sausages decreased contrarily to what happened with the consumption of yoghurts. It highlights the need to tailor food programmes and policies to each segment of the population in order to promote its effectiveness. The present study also showed some differences in food sources of UPF consumption compared to other countries. Although the contributions by age group were not described, in general, in the $\mathrm{UK}^{(8)}$ and in Canada ${ }^{(57)}$, industrialised packaged breads seem to be the highest contributor of UPF consumption. In Portugal, this was not expected because traditionally Portuguese people buy fresh bread at bakeries (considered as processed by the NOVA classification - NOVA group 3). In the Belgian national food consumption survey 2014-2015 ${ }^{(41)}$, milk beverages were the dominated source of UPF among children aged 3-5 years followed by cold meats and sausages. In the aforementioned study, except for children under 5 years old and for girls aged 14-17, cold meats and sausages were the top contributor. Whereas cakes, pies, pastries and the dry cakes and sweet biscuits, and soft drinks were shown to be relevant contributions, mostly in males and younger ages. In Portuguese children, yoghurts, flavoured milks and soft drinks went hand in hand, both having relevant contributions. In adolescents, who consume the highest absolute quantity of UPF, the biggest concern seemed to be the soft drinks.

The differences by sex were greater in adults, where yoghurts were the main contributor in females and soft drinks in males. Dairy products are common in Portugal probably due to greater adherence to the Mediterranean diet compared to other countries. However, over the past 100 years, continuous development and improvement from the ingredients selection to the technological processing techniques brought a huge variety of yoghurts to the market ${ }^{(58)}$. Due to the inherent diversity in the nutrient contents and food matrices of different dairy products, the association between dairy and disease risk has often been contradictory ${ }^{(59)}$. In the present study, unsweetened plain yoghurts were classified as minimally processed foods, plain yoghurts with added sugar as processed and flavoured yoghurts or with artificially sweeteners as ultraprocessed. Focus group studies have made it possible to understand the population's perception of $\mathrm{UPF}^{(60)}$. In a socioeconomically diverse sample from Uruguay, these products were not perceived as harmful to health. Some categories of UPFs were classified as unhealthy (e.g. mayonnaise, potato chips), while others tended to be classified as healthy (e.g. yogurt, granola). For this reason, we suggest food interventions to be based on holistic perspective of foods, promoting food literacy that encompasses nutritional composition and ingredients and thus promoting the consumption of minimally processed yoghurts instead of the UPF alternatives. The industrial reformulation of these yoghurts in order to reduce the use of food additives should also be encouraged. As an attempt to do this to soft drinks production/consumption, in 2017, Portugal introduced a special tax ${ }^{(61,62)}$. Preliminary results of the first year after the tax implementation showed that the industry responded with a reformulation of the products ${ }^{(63)}$. However, the substitution of sugar by additives with a sweetening effect deserves attention ${ }^{(64)}$. Despite different contributors to UPF in different populations, it cannot be directly 
hypothesised that the health effects may be different because of this, so further studies are encouraged to clarify these relations.

There were several strengths and limitations in the present study. Firstly, data from a national representative sample were used in this study, ensuring external validity. In addition, the interviews were carried out by highly trained nutritionists according to standardised procedures and using computerassisted personal interviewing. Data inclusion was easier, systematic and accurate due to the use of the electronic platform, specifically designed for the present project, but following harmonised European procedures ${ }^{(24)}$. Food records and $24 \mathrm{~h}$ recalls methods were listed as those with the best performance in estimating UPF ('high to very high' and 'very high' potential, respectively) as they allow to obtain a high level of detail for each food ${ }^{(65)}$. The multiple-pass dietary interviews minimised the omission of possible forgotten foods. Moreover, this method standardised the level of detail for the description of foods and their quantification, including the portion size estimation by photographs of different portions. Seasonal variability was accounted for since the fieldwork was carried out within 12 months. Dietary assessment included $2 \mathrm{~d}$, evenly distributed through the week ${ }^{(23)}$, despite knowing that non-healthy food consumption appears to be higher on weekends ${ }^{(66)}$. However, an adequate distribution of reports corresponding to weekdays and weekend days was obtained. Still, to give robustness to our estimates, usual intake was modelled using SPADE, in which intra-individual variation is estimated and eliminated.

The use of NOVA classification, widely assumed as a proper classification system based on food processing, also values this work. In general, the studies on UPF consumption and its associated factors currently available use the energy proportion as the outcome. Instead, in this study, the absolute quantity was used whereby comparability with other studies may be affected. However, in order to minimise this limitation, descriptive results on UPF consumption were presented as the relative contribution of UPF both to total energy intake and to total quantity of food. The use of the absolute quantity gives strength to the results since it gives relevance to low or no calorie foods (which have a considerable consumption in Portugal - from $10.6 \%$ of UPF quantity in elderly to 18.5 $\%$ in adults - data not shown or published) while avoiding the residual confounding effect of dividing by energy. The study of UPF through absolute quantity instead of energy will allow us to perceive changes in the consumption of certain foods that have been taxed in the meantime, such as soft drinks. If only considering the energy contribution of UPF, it can be hypothesised that consumption has decreased when in fact it may have been maintained or increased but masked by the use of artificial sweeteners.

\section{Conclusion}

In the Portuguese population aged between 3 and 84 years, the UPF consumption was $313 \mathrm{~g} / \mathrm{d}$ (10.6 \% TQ; $454 \mathrm{kcal} / \mathrm{d} ; 23.8$ $\%$ TEI). Compared to older adults, younger ages were significantly associated with a higher consumption of UPF in both sexes. In adolescents and younger adults, males consumed more UPF than females. Higher education was shown to be positively associated with UPF consumption in adults and negatively in children. Being a single male or a smoking female were other factors that were positively associated with high UPF consumption. Overall, yoghurt-containing additives, soft drinks and cold meats and sausages were the highest contributors to UPF quantity. Yoghurts showed to be the leaders for children and adult females at all levels of education while soft drinks showed to be the leaders in adolescents from both sexes and in younger adults. Cold meats and sausage consumption stood out in males with lower educational levels, decreasing as schooling increases, as opposed to yoghurt consumption. The differences found in these contributors by age group and educational level deserve to be taken into account when designing effective interventions aiming to decrease UPF consumption. However, for some foods such as yoghurts, emphasis should be placed on promoting consumption of the minimally processed ones.

\section{Supplementary material}

The supplementary material for this article can be found at https://doi.org/10.1017/jns.2021.61.

\section{Acknowledgments}

The authors gratefully acknowledge the participants enrolled in the Portuguese National Food, Nutrition and Physical Activity Survey (IAN-AF) 2015-2016 for their kindness and all members of the research team for their enthusiasm.

This article is a result of the project 'UPPer - Consumption of ultra-processed foods, nutrient profile and obesity in Portugal', supported by Competitiveness and Internationalisation Operational Programme (POCI), under the PORTUGAL 2020 Partnership Agreement, through the European Regional Development Fund (ERDF) and through national funds by the FCT - Fundação para a Ciência e a Tecnologia (POCI-01-0145-FEDER-032090 (FCT/FEDER)). This work was also supported by the Fundação de Amparo à Pesquisa do Estado de São Paulo (FAPESP) (grant nos 2019/05972-7, 2018/07391-9 and 2016/14302-7)'. The IAN-AF 2015-2016 has received funding from the EEA Grants Programme, Public Health Initiatives (PT06 000088SI3). This study was financed by national funds through FCT, I.P., under the projects UIDB/04750/2020 and UID/QUI/50006/2020. V. M. benefits from an FCT individual doctoral grant (SFRH/BD/143747/2019).

$\mathrm{V}$. M. conceived the main research questions, analysed and interpreted these data with M. S. and D. C. support. M. S. and D. C. also verified the analytical methods and gave additional inputs to the study design. D. T. and C. L. coordinated the IAN-AF 2015-2016 investigation. R. C. M., F. R. and R. L. revised each draft of the manuscript for important intellectual content. S. R. and R. L. coordinated the UPPer project and had an important role in interpretation of the data. C. L. formulated the main research questions of the present study and supervised it. V. M. wrote the manuscript and all authors discussed the results, contributing to the final document.

There are no conflicts of interest. 


\section{References}

1. Baker P, Machado P, Santos T, et al. (2020) Ultra-processed foods and the nutrition transition: global, regional and national trends, food systems transformations and political economy drivers. Obes Rev 21, e13126.

2. Pan American Health Organization (2015) Ultra-Processed Food and Drink Products in Latin America: Trends, Impact on Obesity, Policy Implications. Washington, DC: World Health Organization.

3. Monteiro CA, Cannon G, Levy RB, et al. (2019) Ultra-processed foods: what they are and how to identify them. Public Health Nutr 22, 936-941.

4. Monteiro CA, Moubarac JC, Cannon G, et al. (2013) Ultra-processed products are becoming dominant in the global food system. Obes Rev 14, 21-28.

5. Djupegot IL, Nenseth CB, Bere E, et al. (2017) The association between time scarcity, sociodemographic correlates and consumption of ultra-processed foods among parents in Norway: a crosssectional study. BMC Public Health 17, 447.

6. Vandevijvere S, Jaacks LM, Monteiro CA, et al. (2019) Global trends in ultraprocessed food and drink product sales and their association with adult body mass index trajectories. Obes Rev 20, $10-19$.

7. Zhang Z, Jackson SL, Martinez E, et al. (2021) Association between ultraprocessed food intake and cardiovascular health in US adults: a cross-sectional analysis of the NHANES 2011-2016. Am J Clin Nutr 113, 428-436.

8. Rauber F, da Costa Louzada ML, Steele EM, et al. (2018) Ultra-processed food consumption and chronic non-communicable diseases-related dietary nutrient profile in the UK (2008-2014). Nutrients 10, 587.

9. Costa de Miranda R, Rauber F, de Moraes MM, et al. (2021) Consumption of ultra-processed foods and non-communicable disease-related nutrient profile in Portuguese adults and elderly (2015-2016): the UPPER project. Br J Nutr 125, 1177-1187.

10. Askari M, Heshmati J, Shahinfar H, et al. (2020) Ultra-processed food and the risk of overweight and obesity: a systematic review and meta-analysis of observational studies. Int J Obes 44, 2080 2091.

11. Pagliai G, Dinu M, Madarena MP, et al. (2021) Consumption of ultra-processed foods and health status: a systematic review and meta-analysis. Br J Nutr 125, 308-318.

12. Santos FSD, Dias MDS, Mintem GC, et al. (2020) Food processing and cardiometabolic risk factors: a systematic review. Rev Saude Publica 54, 70.

13. Chen X, Zhang Z, Yang H, et al. (2020) Consumption of ultraprocessed foods and health outcomes: a systematic review of epidemiological studies. Nutr J 19, 86.

14. Silva Meneguelli T, Viana Hinkelmann J, Hermsdorff HHM, et al. (2020) Food consumption by degree of processing and cardiometabolic risk: a systematic review. Int J Food Sci Nutr 71, 678-692.

15. Moubarac JC, Martins AP, Claro RM, et al. (2013) Consumption of ultra-processed foods and likely impact on human health. Evidence from Canada. Public Health Nutr 16, 2240-2248.

16. Schnabel L, Kesse-Guyot E, Alles B, et al. (2019) Association between ultraprocessed food consumption and risk of mortality among middle-aged adults in France. JAMA Int Med 179, 490-498.

17. Simoes BDS, Barreto SM, Molina M, et al. (2018) Consumption of ultra-processed foods and socioeconomic position: a cross-sectional analysis of the Brazilian Longitudinal Study of Adult Health (ELSA-Brasil). Cad Saude Publica 34, e00019717.

18. Khandpur N, Cediel G, Obando DA, et al. (2020) Sociodemographic factors associated with the consumption of ultra-processed foods in Colombia. Rev Saude Publica 54, 19.

19. Vilela S, Muresan I, Correia D, et al. (2020) The role of socioeconomic factors in food consumption of Portuguese children and adolescents: results from the National Food, Nutrition and Physical Activity Survey 2015-2016. Br J Nutr 124, 591-601.
20. Rauber F, Steele EM, Louzada M, et al. (2020) Ultra-processed food consumption and indicators of obesity in the United Kingdom population (2008-2016). PLoS One 15, e0232676.

21. Costa CDS, Flores TR, Wendt A, et al. (2018) Sedentary behavior and consumption of ultra-processed foods by Brazilian adolescents: Brazilian National School Health Survey (PeNSE), 2015. Cad Saude Publica 34, e00021017.

22. Lopes C, Torres D, Oliveira A, et al. (2018) National food, nutrition, and physical activity survey of the Portuguese general population (2015-2016): protocol for design and development. JMIR Res Protoc 7, e42.

23. Lopes C, Torres D, Oliveira A, et al. (2017) National food, nutrition and physical activity survey of the Portuguese general population. EFSA Support Publ 14, 37. doi: 10.2903/sp.efsa.2017.EN-1341.

24. European Food Safety Authority (2014) Guidance on the EU menu methodology. EFS A J 12, 3944.

25. Goios AC, Severo M, Lloyd AJ, et al. (2020) Validation of a new software eAT24 used to assess dietary intake in the adult Portuguese population. Public Health Nutr 23, 3093-3103.

26. Conway JM, Ingwersen LA, Vinyard BT, et al. (2003) Effectiveness of the US Department of Agriculture 5-step multiple-pass method in assessing food intake in obese and nonobese women. Am J Clin Nutr 77, 1171-1178.

27. European Food Safety Authority (2011) Report on the Development of A Food Classification and Description System for Exposure Assessment and Guidance on its Implementation and Use. EFS A Journal 9, 2489.

28. National Health Institute Doutor Ricardo Jorge (2006) Food composition table. In Portuguese: Tabela da Composição de Alimentos [Center of Food Safety and Nutrition, National Institute of Health Doutor Ricardo Jorge], pp. 1-355. Lisbon: National Health Institute Doutor Ricardo Jorge.

29. Lopes C, Torres D, Oliveira A, et al. (2018) National Food, Nutrition, and Physical Activity Survey of the Portuguese General Population. IAN-AF 2015-2016: Summary of Results; available at www.ian-af.up.pt. ISBN: 978-989-746-202-3.

30. Torres D, Faria N, Sousa N, et al. (2017) Inquérito Alimentar Nacional e de Atividade Física. IAN-AF 2015-2016: Manual Fotográfco de Quantifcação de Alimentos. Universidade do Porto; disponível em: www.ian-af.up.pt. ISBN: 978-989-746182-8 [in Portuguese].

31. Monteiro CA, Cannon G, Moubarac JC, et al. (2018) The UN Decade of Nutrition, the NOVA food classification and the trouble with ultra-processing. Public Health Nutr 21, 5-17.

32. Instituto Nacional de Estatistica (Portugal) (2014) The New Classification of Urban Areas. Deliberation of the Standing Section of Statistical Coordination of the Statistical Council. Available at: https:// www.ine.pt.

33. Bickel G, Nord M, Price C, et al. (2000) Guide to Measuring Household Food Security, Revised 2000. Alexandria, VA: U.S. Department of Agriculture, Food and Nutrition Service.

34. Bassett DR Jr (2003) International Physical Activity Questionnaire: 12-country reliability and validity. Med Sci Sports Exerc 35, 1396.

35. Dekkers AL, Verkaik-Kloosterman J, van Rossum CT, et al. (2014) SPADE, a new statistical program to estimate habitual dietary intake from multiple food sources and dietary supplements. $J$ Nutr 144, 2083-2091.

36. Forshee RA \& Storey ML (2004) Controversy and statistical issues in the use of nutrient densities in assessing diet quality. J Nutr 134, $2733-2737$.

37. Kim HY (2013) Statistical notes for clinical researchers: assessing normal distribution (2) using skewness and kurtosis. Restor Dent Endod 38, 52-54.

38. Core Team R (2017) R: A Language and Environment for Statistical Computing. Vienna, Austria: R Foundation for Statistical Computing; available at https://www.R-project.org/.

39. Lumley T (2020) Survey: Analysis of Complex Survey Samples. R package version 4.0. 
40. Monteiro CA, Moubarac JC, Levy RB, et al. (2018) Household availability of ultra-processed foods and obesity in nineteen European countries. Public Health Nutr 21, 18-26.

41. Vandevijvere S, De Ridder K, Fiolet T, et al. (2019) Consumption of ultra-processed food products and diet quality among children, adolescents and adults in Belgium. Eur J Nutr 58, 3267-3278.

42. Orfanos P, Naska A, Rodrigues S, et al. (2017) Eating at restaurants, at work or at home. Is there a difference? A study among adults of 11 European countries in the context of the HECTOR* project. Eur J Clin Nutr 71, 407-419.

43. Vilarnau C, Stracker DM, Funtikov A, et al. (2019) Worldwide adherence to Mediterranean diet between 1960 and 2011. Eur J Clin Nutr 72, 83-91.

44. Oliveira A, Lopes C \& Rodríguez-Artalejo F (2010) Adherence to the southern European Atlantic diet and occurrence of nonfatal acute myocardial infarction. Am J Clin Nutr 92, 211-217.

45. da Rocha BRS, Rico-Campa A, Romanos-Nanclares A, et al. (2021) Adherence to Mediterranean diet is inversely associated with the consumption of ultra-processed foods among Spanish children: the SENDO project. Public Health Nutr 24, 3294-3303.

46. Blanco-Rojo R, Sandoval-Insausti H, Lopez-Garcia E, et al. (2019) Consumption of ultra-processed foods and mortality: a national prospective cohort in Spain. Mayo Clin Proc 94, 2178-2188.

47. Srour B, Fezeu LK, Kesse-Guyot E, et al. (2019) Ultra-processed food intake and risk of cardiovascular disease: prospective cohort study (NutriNet-Sante). BMJ 365, 11451.

48. Durão C, Severo M, Oliveira A, et al. (2017) Association between dietary patterns and adiposity from 4 to 7 years of age. Public Health Nutr 20, 1973-1982.

49. Northstone K \& Emmett PM (2008) Are dietary patterns stable throughout early and mid-childhood? A birth cohort study. $\mathrm{Br} \mathrm{J}$ Nutr 100, 1069-1076.

50. Cruz F, Ramos E, Lopes C, et al. (2018) Tracking of food and nutrient intake from adolescence into early adulthood. Nutrition 55-56, 84-90.

51. Mikkilä V, Räsänen L, Raitakari OT, et al. (2005) Consistent dietary patterns identified from childhood to adulthood: the cardiovascular risk in young Finns study. Br J Nutr 93, 923-931.

52. Marron-Ponce JA, Sanchez-Pimienta TG, Louzada M, et al. (2018) Energy contribution of NOVA food groups and sociodemographic determinants of ultra-processed food consumption in the Mexican population. Public Health Nutr 21, 87-93.
53. Baraldi LG, Martinez Steele E, Canella DS, et al. (2018) Consumption of ultra-processed foods and associated sociodemographic factors in the USA between 2007 and 2012: evidence from a nationally representative cross-sectional study. BMJ Open 8, e020574.

54. Maia EG, Dos Passos CM, Levy RB, et al. (2020) What to expect from the price of healthy and unhealthy foods over time? The case from Brazil. Public Health Nutr 23, 579-588.

55. Fagerberg P, Langlet B, Oravsky A, et al. (2019) Ultra-processed food advertisements dominate the food advertising landscape in two Stockholm areas with low vs high socioeconomic status. Is it time for regulatory action? BMC Public Health 19, 1717.

56. Lam MCL \& Adams J (2017) Association between home food preparation skills and behaviour, and consumption of ultraprocessed foods: cross-sectional analysis of the UK National diet and nutrition survey (2008-2009). Int J Behav Nutr Phys Act 14, 68.

57. Nardocci M, Leclerc BS, Louzada ML, et al. (2019) Consumption of ultra-processed foods and obesity in Canada. Can J Public Health $110,4-14$.

58. Aryana KJ \& Olson DW (2017) A 100-year review: yogurt and other cultured dairy products. J Dairy Sci 100, 9987-10013.

59. Thorning TK, Bertram HC, Bonjour JP, et al. (2017) Whole dairy matrix or single nutrients in assessment of health effects: current evidence and knowledge gaps. Am J Clin Nutr 105, 1033-1045.

60. Machín L, Antúnez L, Curutchet MR, et al. (2020) The heuristics that guide healthiness perception of ultra-processed foods: a qualitative exploration. Public Health Nutr 23, 2932-2940.

61. Lei n. ${ }^{\circ} 42 / 2016$ de 28 de dezembro. Diário da República n. ${ }^{\circ} 248$ $1 .^{a}$ Série. Lisboa.

62. Lei n. ${ }^{\circ} 71 / 2018$ de 31 de dezembro. Diário da República n..$^{\circ} 251$ $1{ }^{a}$ Série. Lisboa.

63. Baptista S, da Silva FG, Silva D, et al. (2018) Impacto do Imposto Especial sobre o Consumo de Bebidas Açucaradas e Adicionadas de Edulcorantes. Relatório do grupo de trabalho (Despacho $\mathrm{n}^{\circ}$ 2774/2018).

64. Russell C, Grimes C, Baker P, et al. (2020) The drivers, trends and dietary impacts of non-nutritive sweeteners in the food supply: a narrative review. Nutrition Res Rev, 1-24.

65. FAO (2015) Guidelines on the Collection of Information on Food Processing Through Food Consumption Surveys. Rome: FAO.

66. Czlapka-Matyasik M, Lonnie M, Wadolowska L, et al. (2018) 'Cutting down on sugar' by non-dieting young women: an impact on diet quality on weekdays and the weekend. Nutrients 10, 1463. 